\title{
Prediction of lung function and lung density of young adults who had bronchopulmonary dysplasia
}

\author{
Helger Y. Santema ${ }^{1}$, Jan Stolk ${ }^{1}$, Mady Los ${ }^{1}$, Berend C. Stoel ${ }^{2}$, Roula Tsonaka ${ }^{3}$ \\ and Istvan T. Merth ${ }^{4}$
}

Affiliations: ${ }^{1}$ Dept of Pulmonology, Leiden University Medical Center, Leiden, The Netherlands. ${ }^{2}$ Dept of Radiology, Leiden University Medical Center, Leiden, The Netherlands. ${ }^{3}$ Dept of Medical Statistics, Leiden University Medical Center, Leiden, The Netherlands. ${ }^{4}$ Dept of Pediatrics, Ziekenhuisgroep Twente, Almelo, The Netherlands.

Correspondence: Jan Stolk, Dept of Pulmonology, Leiden University Medical Center, P0 box 9600 (C2-R), 2300 RC Leiden, The Netherlands. E-mail: j.stolkalumc.nl

ABSTRACT COPD risk is jointly determined by fetal lung development, lung growth rate and lung growth duration leading to the maximally attained level of lung function in early adulthood. Bronchopulmonary dysplasia (BPD) is considered a developmental arrest of alveolarisation. Long-term outcome studies of adult survivors born before the introduction of surfactant therapy ("old BPD") showed impaired lung function. We aimed to predict adult lung function and lung density in a cohort of premature infants born in the surfactant era, representing "new BPD".

We studied a cohort of young adults born between 1987 and 1998, with $(n=36)$ and without $(n=28)$ $\mathrm{BPD}$, treated in a single centre. Their perinatal characteristics and pulmonary function in infancy were studied by regression analysis for correlation with adult lung function and tissue lung density, all expressed by $z$-scores, at a mean age of $19.7 \pm 1.1$ and $21 \pm 2.2$ years, respectively.

Although BPD adults had on average lower forced expiratory volume in $1 \mathrm{~s}\left(\mathrm{zFEV}_{1}\right) /$ forced vital capacity (FVC) and $\mathrm{zFEV}_{1}$ than those without, $55 \%$ of the BPD group had $\mathrm{zFEV} 1 / \mathrm{FVC}$ values above the lower limit of normal (LLN). Moreover, above LLN values of diffusing capacity of the lung for carbon monoxide $\left(\mathrm{z} D_{\mathrm{LCO}}\right)$ was present in $89 \%$ of BPD adults and lung density in $71 \%$. Only higher oxygen supply $\left(F_{\mathrm{IO} 2}\right)$ at 36 weeks post-conception of $\mathrm{BPD}$ subjects had a trend with lower $\mathrm{ZFEV}_{1}(\mathrm{~B}=-6.4 ; \mathrm{p}=0.053)$ and lower $\mathrm{z} D_{\mathrm{LCO}}(\mathrm{B}=-4.1 ; \mathrm{p}=0.023)$ at adulthood.

No statistically significant predictors of new BPD were identified.

@ERSpublications

Neither perinatal clinical parameters nor infant pulmonary function at 1 year corrected age could predict outcomes of adult lung function and lung tissue density in a cohort of adults who survived BPD https://bit.ly/3gL6gaU

Cite this article as: Santema HY, Stolk J, Los M, et al. Prediction of lung function and lung density of young adults who had bronchopulmonary dysplasia. ERJ Open Res 2020; 6: 00157-2020 [https:// doi.org/10.1183/23120541.00157-2020].

This article has supplementary material available from openres.ersjournals.com

Received: 30 March 2020 | Accepted after revision: 2 Aug 2020

Copyright $\odot$ ERS 2020. This article is open access and distributed under the terms of the Creative Commons Attribution Non-Commercial Licence 4.0 


\section{Introduction}

Bronchopulmonary dysplasia (BPD), also known as neonatal chronic lung disease [1] remains the most common long-term pulmonary complication of prematurely born infants. They often require prolonged respiratory support during the first year of life [2]. Improvements in neonatal respiratory care did not decrease prevalence $[3,4]$. Histological analysis of human and animal lung tissue of BPD showed an arrest of alveolarisation [5, 6], leading to abnormal lung parenchyma [2]. These are reflected by impaired pulmonary function measured already in infancy $[7,8]$. Long-term studies suggest that BPD subjects have decreased forced expiratory volume in $1 \mathrm{~s}\left(\mathrm{FEV}_{1}\right)$, at mid-childhood and early adolescence [9-16], which may become permanent later in life. Airflow limitation may also be due to reduced pulmonary elastic recoil resulting from the disordered development of the acinus. Lung parenchymal changes have been shown in young adult survivors of BPD by computed tomography of the chest [10], and suggest that BPD may be an important risk factor for COPD at adult age [17]. Most studies include young adults with BPD born before surfactant replacement therapy became standard care, classified as "old BPD". Furthermore, these studies are cross-sectional providing no information on their pulmonary function in early life, precluding a better understanding of the natural course of BPD. Clinical trials performed between 1987 and 1994 have proven that surfactant therapy is effective in improving the immediate need for respiratory support and the clinical outcome of premature newborns as reported by a Cochrane analysis in 2000 [18].

The aim of the present study was to define factors of perinatal characteristics and pulmonary function in infancy that predict adult lung function and density in a cohort of young adults born with BPD in the surfactant era ("new BPD”) at Leiden University Medical Centre between 1987 and 1998.

\section{Study subjects}

The Medical Ethics Committee of Leiden University Medical Centre approved this ongoing study and informed parental consent was obtained for the participation of the infants. This cohort was previously described by MerTh et al. [7] and included infants with and without hyaline membrane disease (HMD) during the first year of life. Inclusion of infants to the study population continued until 1998. Eighty-six subjects were enrolled between 1987 and 1998 (figure1). Twenty-four were healthy prematurely (HP) born infants, 19 developed HMD without BPD and 45 were diagnosed as BPD if supplemental oxygen at 36 weeks of gestation was required [19] with typical radiographical changes and chronic dyspnoea [7]. As clinical data of HP and HMD groups revealed no statistically significant differences between them (supplementary material table S1), all data of these two groups were combined to qualify as non-BPD. The perinatal characteristics and characteristics for gestational corrected age (GCA) of 1 year of the participants are summarised in table 1.

The subject characteristics of the cohort at young adulthood are summarised in table 2.

\section{Study design}

At the age of 19 to 25 years, all subjects from our cohort were invited to our outpatient department for an evaluation of their respiratory status. Figure 1 depicts recruitment of the participants. After taking a history of symptoms and a Saint George's Respiratory Questionnaire (SGRQ), we measured pulmonary function and lung density by computed tomography (CT) scan of the chest on a single day. Those born prematurely but with no diagnosis of BPD participated as controls.

\section{Methods}

\section{Respiratory support during the neonatal period}

Mothers received corticosteroids intra-muscularly ante partum if admitted on time to the hospital before delivery. Surfactant (Curosurf ${ }^{\mathrm{TM}}$ ) was administered if indicated. A course of dexamethasone was prescribed to infants at risk to develop BPD, according to a protocol used at the Neonatal Intensive Care Unit. Both the level of maximum positive inspiratory pressures $\left(\mathrm{PIP}_{\max }\right)$ during mechanical ventilation and the fractional concentration of oxygen $\left(F_{\mathrm{IO}_{2}}\right)$ in ambient air at the post-conceptional age of 36 weeks were registered. Oxygen was administered to the spontaneously breathing infant, either in an incubator (Air-Shields C200 (Europe) GmbH, Germany) or in an oxygen tent (Croupette, Air-Shields (Europe) $\mathrm{GmbH}$, Güdingen, Germany). $F_{\mathrm{IO}_{2}}$ was determined by an oxygen concentration analyser (OxyCheck II Model 2015; Critikon $\mathrm{GmbH}$, Norderstedt, Germany) positioned next to the infant's face. The $F_{\mathrm{IO}_{2}}$ was tailored to achieve transcutaneous $\mathrm{O}_{2}$ saturation (Pulse Oximeter, Type 1.1.9.0; Nellcor Inc, Hayward, CA, USA) in the range $92-98 \%$ (table 1). Four patients received oxygen supplementation through a nasopharyngeal cannula [20]. However, their $F_{\mathrm{IO}_{2}}$ could not be defined accurately, hence they have been excluded from the multiple regression analysis. 
TABLE 1 Perinatal characteristics and at gestational corrected age (GCA) of 1 year

\begin{tabular}{|c|c|c|c|c|}
\hline Male & $18 / 28(64 \%)$ & $24 / 36(67 \%)$ & 0 & 0.84 \\
\hline Weeks of pregnancy & $31.0 \pm 2.6$ & $28.2 \pm 2.3$ & 0 & $<0.0001$ \\
\hline Birth weight percentiles & $52.5 \pm 29.6$ & $51.6 \pm 31.3$ & 0 & 0.91 \\
\hline Surfactant therapy & $11 / 28(39 \%)$ & $21 / 36(58 \%)$ & 0 & 0.13 \\
\hline Prenatal dexamethasone ${ }^{\#}$ & $7 / 28(25 \%)$ & $16 / 36(44 \%)$ & 0 & 0.11 \\
\hline $\mathrm{PIP}_{\max }$ & $8.2 \pm 10.9$ & $24.7 \pm 7.5$ & $5 / 5$ & $<0.0001$ \\
\hline$F_{1 O_{2}}$ at 36 weeks & 0.21 & $0.29 \pm 0.08$ & $6 / 0$ & $<0.0001$ \\
\hline GCA at lung function testing months & $9.7 \pm 3.8$ & $10.4 \pm 3.0$ & $1 / 1$ & 0.47 \\
\hline $\mathrm{CHL}$ at lung function testing $\mathrm{cm}$ & $71 \pm 9$ & $71 \pm 7$ & $2 / 1$ & 0.73 \\
\hline FRC z-score for CHL & $0.05 \pm 1.031$ & $0.37 \pm 1.31$ & $2 / 1$ & 0.30 \\
\hline Compliance z-score for CHL & $0.32 \pm 1.03$ & $-0.56 \pm 0.82$ & $3 / 1$ & 0.0005 \\
\hline \multicolumn{5}{|c|}{  } \\
\hline
\end{tabular}

Lung function at the age of 1 year, corrected for gestational age

Infant pulmonary function tests included functional residual capacity, respiratory system compliance and mixing efficiency as described previously [7]. Results presented in the publication by MerTH et al. [7] were supplemented with subjects identified thereafter. Details are shown in table 1.

\section{Lung function and density at young adulthood}

Each subject had all assessments at adult age performed on the same day. Lung function was assessed according to the ATS/ERS recommendations [21, 22], using Jaeger Masterscreen PFT-Pro and Jaeger Masterscreen CS functional residual capacity (FRC). About 15 min before spirometry, all participants had inhaled $400 \mu \mathrm{g}$ salbutamol. Results of spirometry $\left(\mathrm{FEV}_{1}, \mathrm{FVC}\right)$, lung volumes (FRC, residual volume (RV), total lung capacity (TLC)), using helium dilution and CO diffusing capacity, using the single-breath method, are expressed as z-scores obtained by prediction equations, as recommended [23, 24]. In three BPD subjects the flow-volume curves of spirometry did not meet the European Respiratory Society/ American Thoracic Society quality criteria and were excluded from further analysis. The same applied to the diffusing capacity of the lung for carbon monoxide $\left(D_{\mathrm{LCO}}\right)$ measurement of one BPD subject. The status of the terminal airways reflected by ventilation distribution was assessed by the mixing efficiency (ME), calculated from the helium wash-in curve as the ratio of the ideal to the actual number of breaths to achieve $90 \%$ of the final helium concentration [25]. In one subject of the BPD group, this test could not be applied. To assess parenchymal alveolar tissue, lung density was quantified by CT scanning of the chest without contrast enhancement (Toshiba Aquilion, 64 detector rows, with the following scan protocol: $135 \mathrm{kVp}, 40 \mathrm{~mA}$, rotation time $0.5 \mathrm{~s}$, collimation $64 \times 0.5 \mathrm{~mm}$, detector pitch 1.5 ; reconstruction kernel

TABLE 2 Subject characteristics at adulthood

\begin{tabular}{|c|c|c|c|c|}
\hline & Non-BPD group ( $n=28$ ) & BPD group ( $n=36)$ & Missing & p-value \\
\hline Smoking & $4 / 28(14 \%)$ & $7 / 36(19 \%)$ & 0 & 0.74 \\
\hline Age at lung function testing years & $21.0 \pm 2.5$ & $19.7 \pm 1.1$ & 0 & 0.0077 \\
\hline Weight kg & $68 \pm 12$ & $69 \pm 19$ & 0 & 0.89 \\
\hline Length $\mathrm{cm}$ & $177 \pm 10.3$ & $174 \pm 10$ & 0 & 0.21 \\
\hline BMI $\mathrm{kg} \cdot \mathrm{m}^{-2}$ & $21.7 \pm 2.8$ & $22.6 \pm 4.9$ & 0 & 0.38 \\
\hline
\end{tabular}

Data are presented as mean \pm SD or $\mathrm{n} / \mathrm{N}(\%)$, unless otherwise stated. BPD: bronchopulmonary dysplasia; Smoking: ever-smoking (yes=1, no=0); BMl: body mass index $\mathrm{kg} \cdot \mathrm{m}^{-2}$. 


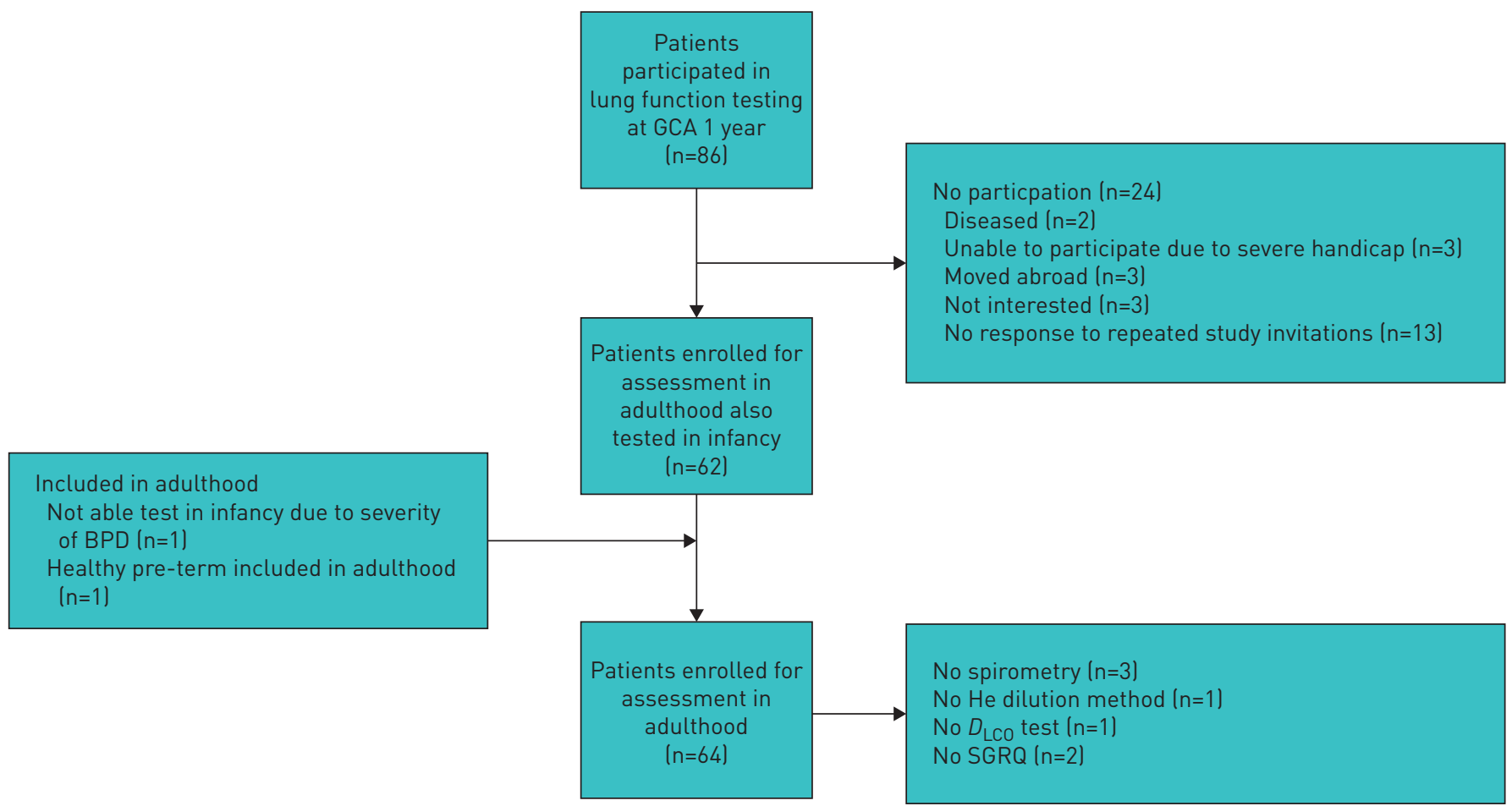

FIGURE 1 Patient disposition. Flow chart of the participants. Seven healthy prematurely (HP) born infants could not be located for the study. However, one subject, who was not measured in infancy, could be included in the adult group. Three refused to participate and one moved abroad leaving $14 \mathrm{HP}$ participants. Of the hyaline membrane disease (HMD) subjects, 5 could not be located at adult age leaving 14 HMD participants. Of those with bronchopulmonary dysplasia (BPD), 2 passed away during infancy, 2 could not be located, 2 have moved abroad, 3 refused to participate and 3 were severely mentally handicapped, precluding them to participate at adulthood leaving 36 BPD participants. As clinical data of $\mathrm{HP}$ and HMD groups revealed no differences (supplementary table S1), all data of these two groups were combined to qualify as non-BPD (non-BPD, n=28). Thus, in total, 28 non-BPD served as controls and 36 BPD subjects were analysed in the study. All HP subjects were White except for one from Indonesia and two from North African, one HMD and one BPD subject was from Indonesia.

FC12, slice thickness $5 \mathrm{~mm}$, with increment $2.5 \mathrm{~mm}$ ). The resulting images were analysed by PULMO software (version 2.2. Medis Specials, Leiden, the Netherlands), to calculate the total lung volume and the 15th percentile lung density $\left(\mathrm{PD}_{15}\right)$. The $\mathrm{PD}_{15}$ values were automatically compared with a database of 76 healthy subjects with normal lung function and CT appearance, resulting in a so-called standard mass score (SMS), defined as the z-score for the volume-corrected normal lung density [26].

Respiratory symptoms at adult age were scored by the SGRQ. A score of 22.62 was considered the 95th percentile of normal [27]. Two subjects (one BPD and one HP) did not fill in the questionnaire.

\section{Statistical analysis}

We used IBM SPSS Statistics version 25 software (IBM, Armonk, USA). Categorical clinical variable differences between groups of baseline data have been tested with Chi-squared test and ANOVA has been used for the numerical clinical variables. ANCOVA was used to test differences between the BPD and non-BPD group, while adjusting for additional numeric variables. All variables were plotted to verify normal distribution. Homogeneity of variances between the two groups was tested by Levene's test. We analysed covariates "ever-smoking" and percentile birth weight after statistic verification of independence of the covariates and testing homogeneity of regression slopes. Outcomes calculated as significantly impaired at adult age in the BPD group compared to the non-BPD group were selected and further studied to identify predictors of BPD outcome at adulthood. Univariate linear regression models were used to determine whether values of pulmonary function at GCA 1 year predict impairment of these parameters at adult age of the BPD group. Next, we used multiple linear regression analyses to determine whether perinatal parameters correlated with selected outcomes at adult age within the BPD group. Four perinatal parameters were empirically chosen on best presumed correlation with lung function and structure at adult age. In multiple regression, imputation was used for missing data. However, $F_{\mathrm{IO}_{2}}$ at 36 weeks could not be imputed, as four subjects received another method of oxygen administration (i.e. nasopharyngeal cannula) because of their improved clinical condition, still fulfilling the criteria for BPD (supplementary material figure S1). Two patients had a missing $F_{\mathrm{IO}_{2}}$ value at 36 weeks. In all analyses Bonferroni correction was used to correct for multiple testing. 


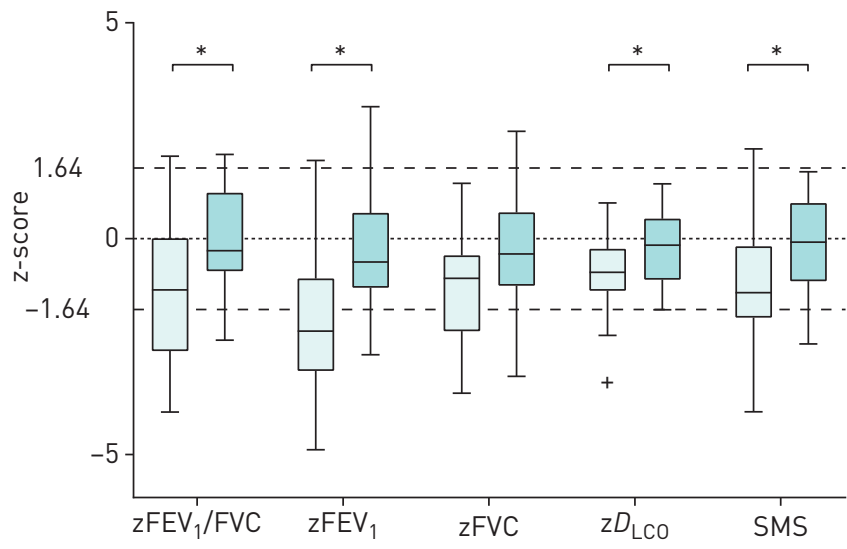

FIGURE 2 Spirometry, gas exchange and lung density at young adulthood. Box plots for lung function parameters of 28 non-BPD subjects (dark box) and 36 BPD subjects (light box) who had spirometry, gas transfer and lung density measured. *: significant difference between groups after correction for birth weight and smoking history $(p<0.005) ; .+$ : outlier.

\section{Results}

The characteristics of study subjects at birth and at for GCA of 1 year are shown in table 1 . Subjects with BPD had a shorter gestational age: $28.2 \pm 2.3$ (mean \pm SD) weeks and a lower birth weight: $1059 \pm 334 \mathrm{~g}$, than those in the non-BPD group: $31.0 \pm 2.6$ weeks and $1484 \pm 440 \mathrm{~g}$, respectively. The mean percentile birth weight was not significantly different. There was a male predominance in both groups: $67 \%$ and $64 \%$, respectively. Mean z-score of compliance and values of ME were significantly lower at 1-year GCA in the BPD infants than in the non-BPD infants. At follow-up as young adults, BPD subjects were examined at a mean age of $19.7 \pm 1.1$ years and those without BPD at a mean age of $21 \pm 2.2$ years (table 2 ).

Young adults with a history of BPD had on average significantly lower $\mathrm{zFEV}_{1}$ and $\mathrm{zFEV} / \mathrm{FVC}$, (figure 2), (supplementary table S2) than non-BPD adults. However, 18 of 33 subjects in the BPD group (55\%) had a $\mathrm{zFEV}_{1} / \mathrm{FVC}$ above the lower limit of normal (LLN), of whom 16 had a $\mathrm{zFEV}_{1}$ (figure 3) above the LLN, while those with a reduced $\mathrm{zFEV}_{1} / \mathrm{FVC}$ ( $\mathrm{n}=15$ of 33 ) had a mean $\mathrm{zFEV}_{1}$ of $-3.09,(-4.89$ to -1.22 , min to max). In the non-BPD group only three subjects had a $z F E V_{1} / F V C$ below the LLN, of whom two had airflow limitation $\left(\mathrm{zFEV}_{1}:-1.67\right.$ and -1.68$)$.

Static lung volumes were not statistically significantly different between the two groups, neither as infants nor as young adults (supplementary table S2). BPD adults had on average a significantly lower diffusing capacity $\left(\mathrm{z} D_{\mathrm{LCO}}\right)$ and lung density (SMS) than those of non-BPD. Nonetheless, a significant number of BPD adults had gas transfer $(n=32 / 36)$ and lung density $(n=25 / 35)$ above the LLN. The BPD group had a significantly higher SGRQ score, than subjects without BPD (supplementary table S2), although both scores were well within the normal range. Three of the BPD subjects had clinically relevant symptoms, whereas none of those without BPD.

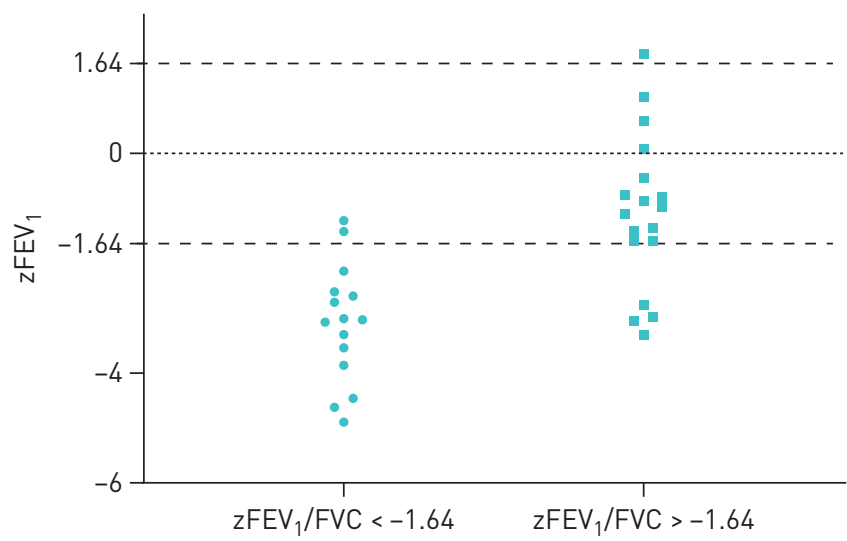

FIGURE 3 Distribution of forced expiratory volume in $1 \mathrm{~s}\left(\mathrm{ZFEV}_{1}\right)$ with and without airway obstruction in the bronchopulmonary dysplasia (BPD) group. In the BPD group (55\%) had a normal $z \mathrm{FEV}_{1} /$ forced vital capacity (FVC) (defined by z-score >-1.64), 16 of whom had a $z F E V_{1}$ above the lower limit of normal (LLN), while those with a $z F E V_{1} / F V C$ below the LLN ( $n=15$ of 33 ) had a mean $z F E V_{1}$ of -3.09 , ( -4.89 to -1.22 , min to max). 


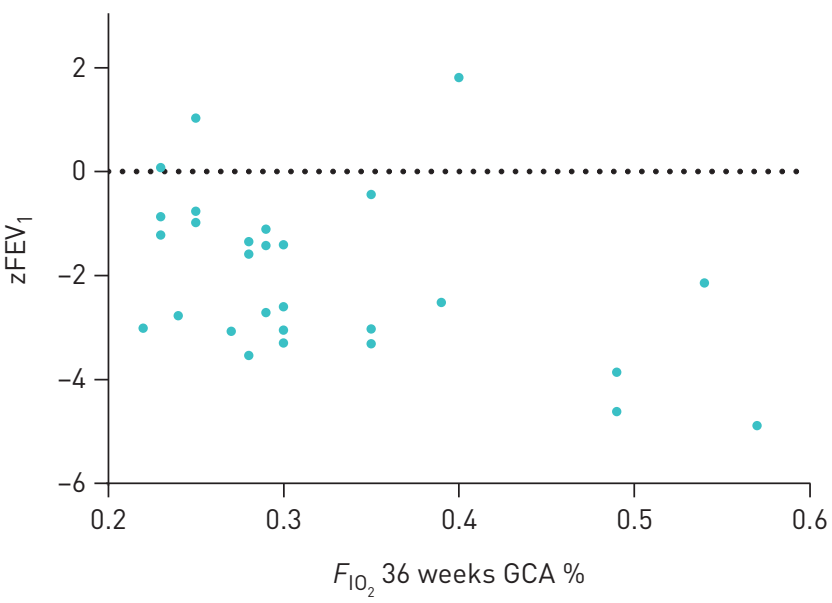

FIGURE 4 Relation between inspiratory oxygen fraction $\left(F_{I_{2}}\right)$ at 36 weeks of gestational corrected age (GCA)

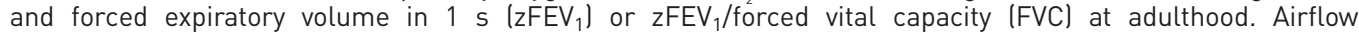
limitation $\left(z \mathrm{FEV}_{1}\right)$ and airway obstruction $\left(z \mathrm{FEV}_{1} / \mathrm{FVC}\right)$ in $29 \mathrm{NCLD}$ adult subjects in relation to the $F_{\mathrm{IO}_{2}}$ at 36 weeks after conception measured while treated with oxygen administered to the spontaneously breathing infant, either in an incubator or in an oxygen tent. Regression analysis for $z F E V_{1}$ revealed $B=-6.4$, with $\mathrm{p}=0.053$, and $\mathrm{B}=-3,2$, with $\mathrm{p}=0.29$ for $\mathrm{ZFEV}_{1} / \mathrm{FVC}$.

Multiple regression analysis using four perinatal factors of BPD subjects revealed that $\mathrm{zFEV} / \mathrm{FVC}$ of $\mathrm{BPD}$ adults tended to be predicted by birth weight $(B=-0.0023 ; \mathrm{p}=0.0091)$. BPD subjects with the highest need for supplemental oxygen $\left(F_{\mathrm{IO}_{2}}\right)$ at 36 weeks after conception tended to have the most severe airflow limitation and the lowest $\mathrm{zFEV}_{1} / \mathrm{FVC}$ (figure 4) at young adult age, although this was not statistically significant after correction for multiple testing. The adjusted p-values of the likelihood ratio test after multiple testing correction are shown in table 3.

Furthermore, univariate linear regression analysis showed that pulmonary function in infancy was neither statistically significantly associated with respiratory function and lung density nor with respiratory symptoms in young adults with a history of BPD (supplementary table S3).

\section{Discussion}

To our knowledge, this is one of few reports currently available on outcome of respiratory function, including spirometry, gas transfer and lung volumes, combined with quantitative lung tissue analysis at young adulthood of infants with BPD who were prematurely born in the surfactant era. Moreover, results of their pulmonary function in infancy are included for the first time in such a study. In contrast to previous reports $[12-14,16]$, our BPD subjects represent the "new BPD" [2]. We hypothesised that outcomes at young adulthood could be predicted by analysis of perinatal characteristics or pulmonary function in infancy, with measurements at an age when the lungs are close to having reached maximal growth in the same individual. This was, however, not born out of our findings.

\section{TABLE 3 Regression analysis of perinatal parameters on outcomes in adult BPD}



Multiple linear regression analyses were used to determine whether neonatal parameters correlated with outcomes at adult age within the $\mathrm{BPD}$ group. None of the dependent variables at adulthood reached a significance level, set at $p<0.0025$ by Bonferroni correction. $z F E V / F V C$, $z F E V_{1}, z F V C$, are $z$-scores for adult lung function outcomes with GLI-references and $z D_{\text {Lco }}$ with European Community of Coal and Steel references. Prenatal dexa: ante partum dexamethasone administered to mothers (yes=1, no=0). BPD: bronchopulmonary dysplasia; FEV ${ }_{1}$ : forced expiratory volume in $1 \mathrm{~s}$; FVC: forced vital capacity; SMS: standard mass score for lung density; SGRQ: St. George's Respiratory Questionnaire; $F_{1 O_{2}} 36 \mathrm{~W}$ : fractional inspired oxygen concentration in ambient air at post-conceptional age of 36 weeks; PIP max: maximum positive inspiratory pressure during mechanical ventilation in $\mathrm{cmH}_{2} \mathrm{O} ; D_{\mathrm{LCO}}$ : diffusing capacity of the lung for carbon monoxide. 
Although young adults with BPD had on average lower airflow, gas transfer and lung density than those without, these parameters turned out to be above the LLN in a significant number of them, suggesting recovery of pulmonary functional impairment in a reasonable percentage of survivors of BPD. The repair of the lungs after BPD occurred despite the use of dexamethasone in $56 \%$ of our BPD subjects during the postnatal period (table 1). Dexamethasone treatment is a well-recognised concern as to its potential to impede lung growth [28]. Apparently, this is not necessarily happening in all individuals receiving the drug who survive neonatal lung injury. Of more concern are the BPD subjects in our cohort with on average significantly lower mean $\mathrm{zFEV}_{1}$, lower $\mathrm{zFEV}_{1} / \mathrm{FVC}$, more impaired $\mathrm{z} D_{\mathrm{LCO}}$ and reduced lung density (SMS), respectively, compared to our non-BPD subjects, at such a young age, being consistent with previous reports $[9,11,16]$. Most of the BPD subjects, in contrast to that described during the early years of childhood [2], had relatively few respiratory symptoms at adult age. Nevertheless, this could worsen during the decades ahead of them.

The percentage of BPD adults we observed with lung function and lung tissue values above the LLN seem to contradict reports of lung function impairment in most adults surviving BPD [9-16]. However, part of these studies $[9,12,14,16]$ included only subjects resembling the "old BPD", characterised by a different pathology and pathophysiology [29] than that described after the introduction of surfactant replacement [30]. While more recent reports included adults representing the "new BPD", they made up only a small part of the whole group born before the surfactant era, hence their results may have been concealed [10, 11, 13], except for the BPD group published by Volsaeter et al. [15] and by Doyle et al. [31]. VolsaETER et al. [15] found in 12 of 26 subjects with "new BPD" a significantly reduced $\mathrm{zFEV}_{1}$ at a mean age of $17.8 \pm 0.7$ years. Doyle et al. [31] found in 164 subjects who were born extremely premature or born with extremely low birth weight that 65 patients had a diagnosis of "new BPD". At 25 years old, only 22 of them had expiratory airflows below the fifth percentile. Our results show percentages similar to VOLSAETER et al. and Doyle et al.

Airflow limitation at young adult age following BPD is considered a long-term sequel of that measured in infancy [8]. Respiratory system resistance was high in our cohort during infancy, as their respiratory system compliance (which is reciprocally related to resistance) was low (table 1) [7]. However, approximately $55 \%$ of subjects of our BPD group had no longer airway obstruction. A significantly reduced $\mathrm{FEV}_{1}$ in the remaining $45 \%$, on the other hand, reflects probably a loss of tethering of the terminal airway walls at their most distal end of the bronchial tree because of reduced pulmonary elastic recoil [2]. This is suggested by a significantly decreased lung density frequently present in early-stage emphysema [32], rendering them at risk for developing COPD [17]. Moreover, a decreased alveolar surface area of the alveolarisation arrest in $\mathrm{BPD}[2,6,30]$ may also have led to decreased $\mathrm{z} D_{\mathrm{LCO}}$ and lung density.

The severity of BPD is graded by the level of supplemental oxygen required at 36 weeks post-conception [2]. We hypothesised that the $\mathrm{F}_{\mathrm{IO}_{2}}$ at 36 weeks would correlate with outcomes at adult age. Those with the highest oxygen need at week 36 tended to have the lowest $\mathrm{ZFEV}_{1}$ (figure 4), although this was not statistically significant after correction for multiple testing. Smoking may have contributed to lower airflows and decreased lung density, although it is unclear to what extent this occurred, because study subjects could not provide the number of pack-years.

A decreased compliance (in combination with normal FRC) and maldistribution of ventilation $[7,8]$, remained the most abnormal findings by the end of the first year of life in most of our BPD infants [7]. These outcomes correspond to the structural changes found in infants who succumbed to BPD [6, 30]. Persistence of such structural parenchymal changes appeared, however, only in a small number of young adult BPD subjects shown by the zRV/TLC and SMS (figure 2).

In contrast to previous studies which combined lung function and imaging by CT scanning of the lungs of young adults surviving BPD $[9,11,16]$, we assessed lung tissue by chest CT-derived lung density [26]. The latter provides a quantitative score, whereas a radiological score is qualitative or semi-quantitative. Given these findings, we consider BPD as one of the contributing factors to COPD in a subgroup of young adults surviving the disease $[15,17,33]$. However, as indicated above, none of the perinatal characteristics could predict in whom COPD occurs at young adulthood. Except for subject heterogeneity and a low power due to relatively small sample size, we cannot explain this.

The reason why lung function and lung density did not improve in about 45\% of the BPD cohort in our study is unclear. We expect that this category of adults will further decline with their lung function later in adulthood [4]. COPD risk is jointly determined by fetal lung development, lung growth rate and lung growth duration leading to the maximally attained level of lung function in early adulthood ("the plateau"), as well as the timing and rate of decline of lung function later in life [34]. Clearly, further research using longitudinal studies of larger cohorts of BPD infants with "new BPD" is needed to 
determine the role of BPD and the novel strategies to alleviate BPD on lung growth and long-term respiratory health in adulthood [4].

The strength of our study is the prospective character of a cohort conducted in a single centre providing availability of detailed perinatal parameters (such as $F_{\mathrm{IO}_{2}}$ ) and unique infant pulmonary function results at GCA of 1 year as starting points for determining the natural course of young adults surviving BPD. Commitment of parents resulted in a high rate of participation of those with BPD (figure 1).

The limitation of our study is, besides the relatively small number of subjects, the lack of data on reversibility of pre-bronchodilator airway calibre. However, the aim of lung function assessment was to detect COPD, not bronchial asthma. The GOLD Guideline for COPD defines the diagnosis as the presence of a post-bronchodilator $\mathrm{FEV}_{1} / \mathrm{FVC}<0.70$ to confirm consistent airflow limitation (www.goldcopd.org). Exercise capacity of our study subjects would have provided more information about gas transfer. We preferred to maximise patient participation by limiting the individual testing sessions to $3 \mathrm{~h}$. Hence, we limited our study protocol to what is presented here. Several BPD subjects in our cohort were not able to perform the tests properly because of some degree of mental disability, a well-known long-term sequel of this group of patients [2], which reduced the power of our results. There was a limited willingness of those who did not develop BPD to participate, probably because of lack of pulmonary symptoms and this may have introduced a selection bias.

In conclusion, neither perinatal characteristics, nor infant pulmonary function at 1-year corrected age could predict outcomes of adult lung function and density in this cohort of young adults who survived BPD. In approximately $45 \%$ of young adults who survived BPD, we observed airflow limitation, decreased gas transfer and decreased lung density, rendering them at risk of developing COPD. However, lung function and lung density was above the LLN in a significant sample of adult BPD survivors born after the introduction of surfactant replacement therapy. These findings suggest that generation of normal peripheral lung tissue may take place in the course to adulthood after premature birth with BPD [35].

Acknowledgements: We wish to acknowledge the late Prof. P. Quanjer for his support and dedication to this project right from the very beginning. His advisory role ended abruptly during the period of discussions of the findings in 2017 because of his untimely death. We admired his invaluable contribution to this study.

Conflict of interest: None declared.

Support statement: Financial support for the study was provided by Stichting Alphal International Registry. The sponsor had no role in the conduct and reporting of the study. Funding information for this article has been deposited with the Crossref Funder Registry.

\section{References}

1 Northway WH, Jr., Rosan RC, Porter DY. Pulmonary disease following respirator therapy of hyaline-membrane disease. Bronchopulmonary dysplasia. N Engl J Med 1967; 276: 357-368.

2 Kinsella JP, Greenough A, Abman SH. Bronchopulmonary dysplasia. Lancet 2006; 367: 1421-1431.

3 Jensen EA, Schmidt B. Epidemiology of bronchopulmonary dysplasia. Birth Defects Res Part A Clin Mol Teratol 2014; 100: 145-157.

4 Bancalari E, Jain D. Bronchopulmonary dysplasia: 50 years after the original description. Neonatology 2019; 115: 384-391.

5 Coalson JJ, Winter V, deLemos RA. Decreased alveolarization in baboon survivors with bronchopulmonary dysplasia. Am J Respir Crit Care Med 1995; 152: 640-646.

6 Margraf LR, Tomashefski JF, Jr, Bruce MC, et al. Morphometric analysis of the lung in bronchopulmonary dysplasia. Am Rev Respir Dis 1991; 143: 391-400.

$7 \quad$ Merth IT, de Winter JP, Zonderland HM, et al. Pulmonary function in infants with neonatal chronic lung disease with or without hyaline membrane disease at birth. Eur Respir J 1997; 10: 1606-1613.

8 Tepper RS, Morgan WJ, Cota K, et al. Expiratory flow limitation in infants with bronchopulmonary dysplasia. J Pediatr 1986; 109: 1040-1046.

9 Aquino SL, Schechter MS, Chiles C, et al. High-resolution inspiratory and expiratory CT in older children and adults with bronchopulmonary dysplasia. AJR Am J Roentgenol 1999; 173: 963-967.

10 Aukland SM, Rosendahl K, Owens CM, et al. Neonatal bronchopulmonary dysplasia predicts abnormal pulmonary HRCT scans in long-term survivors of extreme preterm birth. Thorax 2009; 64: 405-410.

11 Caskey S, Gough A, Rowan S, et al. Structural and functional lung impairment in adult survivors of bronchopulmonary dysplasia. Ann Am Thorac Soc 2016; 13: 1262-1270.

12 Doyle LW, Faber B, Callanan C, et al. Bronchopulmonary dysplasia in very low birth weight subjects and lung function in late adolescence. Pediatrics 2006; 118: 108-113.

13 Gough A, Linden M, Spence D, et al. Impaired lung function and health status in adult survivors of bronchopulmonary dysplasia. Eur Respir J 2014; 43: 808-816.

14 Northway WH J, Moss RB, Carlisle KB, et al. Late pulmonary sequelae of bronchopulmonary dysplasia. $N$ Engl J Med 1990; 323: 1793-1799.

15 Vollsaeter M, Roksund OD, Eide GE, et al. Lung function after preterm birth: development from mid-childhood to adulthood. Thorax 2013; 68: 767-776.

16 Wong PM, Lees AN, Louw J, et al. Emphysema in young adult survivors of moderate-to-severe bronchopulmonary dysplasia. Eur Respir J 2008; 32: 321-328. 
Martinez FD. Early-life origins of chronic obstructive pulmonary disease. N Engl J Med 2016; 375: 871-878.

Soll RF, Morley CJ. Prophylactic versus selective use of surfactant for preventing morbidity and mortality in preterm infants. Cochrane Database Syst Rev 2000; 2: CD000510.

oxygen requirement in the neonatal period. Pediatrics 1988; 82: 527-532.

Shann F, Gatchalian S, Hutchinson R. Nasopharyngeal oxygen in children. Lancet 1988; 2: 1238-1240.

Miller MR, Hankinson J, Brusasco V, et al. Standardisation of spirometry. Eur Respir J 2005; 26: 319-338.

Stanojevic S, Graham BL, Cooper BG, et al. Official ERS technical standards: Global Lung Function Initiative reference values for the carbon monoxide transfer factor for Caucasians. Eur Respir J 2017; 50: 1700010.

Quanjer PH, Hall GL, Stanojevic S, et al. Age- and height-based prediction bias in spirometry reference equations. Eur Respir J 2012; 40: 190-197.

Laszlo G. European standards for lung function testing: 1993 update. Thorax 1993; 48: 873-876.

Bates DV, Christie RV. Intrapulmonary mixing of helium in health and in emphysema. Clin Sci 1950; 9: 17.

Stoel BC, Stolk J, Bakker ME, et al. Regional lung densities in $\alpha-1$ antitrypsin deficiency compared to predicted values. Respir Res 2019; 20: 45.

Ferrer M, Villasante C, Alonso J, et al. Interpretation of quality of life scores from the St George's Respiratory Questionnaire. Eur Respir J 2002; 19: 405-413.

8 Jobe AH. Postnatal corticosteroids for preterm infants - Do what we say, not what we do. N Engl J Med 2004; 350: 1349-1351.

9 Stocker JT. Pathologic features of long-standing "healed" bronchopulmonary dysplasia: a study of 28 3- to 40-month-old infants. Hum Pathol 1986; 17: 943-961.

Husain AN, Siddiqui NH, Stocker JT. Pathology of arrested acinar development in postsurfactant bronchopulmonary dysplasia. Hum Pathol 1998; 29: 710-717.

Doyle LW, Irving L, Haikerwal A, et al. Airway obstruction in young adults born extremely preterm or extremely low birth weight in the postsurfactant era. Thorax 2019; 74: 1147-1153.

Pompe E, Galban CJ, Ross BD, et al. Parametric response mapping on chest computed tomography associates with clinical and functional parameters in chronic obstructive pulmonary disease. Respir Med 2017; 123: 48-55. Baraldi E, Filippone M. Chronic lung disease after premature birth. N Engl J Med 2007; 357: 1946-1955.

Milanzi EB, Koppelman GH, Smit HA, et al. Timing of secondhand smoke, pet, dampness or mould exposure and lung function in adolescence. Thorax 2020; 75: 153-163.

5 Tata PR, Rajagopal J. Plasticity in the lung: making and breaking cell identity. Development 2017; 144: 755-766. 